\title{
ESQUEMA EXPLÍCITO PARA LA SOLUCIÓN NUMÉRICA DEL FLUJO NO SATURADO EN MEDIOS HETEROGÉNEOS BAJO CONDICIÓN DE NIVELES FREÁTICOS SOMEROS.
}

\section{EXPLICIT SCHEME FOR THE NUMERICAL SOLUTION OF UNSATURATED FLOW IN HETEROGENEOUS MEDIA UNDER SHALLOW WATER TABLE CONDITIONS.}

\author{
ErikZimmermann $^{(1)(2)}$ \\ ${ }^{(1)}$ Consejo Nacional de Investigaciones Científicas y Técnicas. Buenos Aires, Argentina. \\ ${ }^{(2)}$ Centro Universitario Rosario de Investigaciones Hidroambientales (CURIHAM). Facultad de Ciencias \\ Exactas, Ingenieríay Agrimensura, Universidad Nacional de Rosario. Rosario, Santa Fe, Argentina
}

e-mail: erikz@fceia.unr.edu.ar

\section{RESUMEN}

Se propone un esquema numérico explícito de 4 celdas (4C) para la integración de las ecuaciones de flujo en la zona no saturada diseñado para áreas de llanura con niveles freáticos someros. El esquema contempla un coeficiente de ponderación para definir la conductividad hidráulica no saturada representativa entre celdas adyacentes y considera la heterogeneidad de cada horizonte edáfico. El esquema $4 \mathrm{C}$ es comparado con estimaciones realizadas por el HYDRUS-1D en dos grupos de ensayos modelados, uno con saturación de superficie durante 10 días y otro con series de precipitaciones durante 96 días. Los ensayos numéricos se hicieron para 14 tipos de asociaciones de suelos características de la zona de estudio ( $\mathrm{A}^{\circ}$ del Azul, Bs. As.) y 6 profundidades freáticas (entre 300 y $50 \mathrm{~cm}$ ). Para el primer ensayo, la respuesta del esquema se considera aceptable con diferencias porcentuales del orden al 19\%. En el segundo, el esquema propuesto estimó satisfactoriamente las recargas freáticas con diferencias porcentuales del $1 \%$ entre ambos esquemas. Complementariamente, mostró un muy buen ajuste en las evoluciones de contenidos de humedad de cada estrato. Estos resultados validan el esquema simplificado propuesto permitiendo una respuesta rápida en términos de tiempo computacional.

Palabras clave:esquema numérico explícito, flujo no saturado, llanura, freática somera.

\begin{abstract}
An explicit 4-cell (4C) numerical scheme is proposed for the integration of the flow equations in the unsaturated zone designed for plain areas with shallow water tables. The scheme contemplates a weighting coefficient to define the representative unsaturated hydraulic conductivity between adjacent cells and considers the heterogeneity of each edaphic horizon. The $4 \mathrm{C}$ scheme is compared with estimates made by HYDRUS-1D in two sets of modeled tests, one with surface saturation for 10 days and the other with rainfall series for 96 days. The numerical tests were performed for 14 types of soil associations characteristic of the study area $\left(\mathrm{A}^{\circ}\right.$ del Azul, Bs. As.) and 6 phreatic depths (between 300 and $50 \mathrm{~cm}$ ). For the first trial, the response of the scheme is considered acceptable with percentage differences of about $19 \%$. In the second, the proposed scheme estimated satisfactorily the phreatic recharge with percentage differences of $1 \%$ between both schemes. In addition, it showed a very good adjustment in the evolution of moisture contents of each stratum. These results validate the proposed simplified scheme allowing a fast response in terms of computational time.
\end{abstract}

Keywords: explicit scheme, unsaturated flow, plain, shallow water table. 


\section{INTRODUCCION}

Los avances de la Hidrología Clásica se han desarrollado bajo el concepto de cuenca hidrográfica convencional, en el cual se define claramente un área de aporte, una organización de la red de drenaje y puede identificarse cada componente de la red con un orden de jerarquía. Son los denominados Sistemas Hidrológicos Típicos (SHT).

El concepto clásico de cuenca, en sentido estricto, refleja sólo una parte de los posibles sistemas hidrológicos reales. La expansión del conocimiento a nivel mundial enfrenta a los hidrólogos con las grandes llanuras de muy baja pendiente en las cuales no se manifiesta una red de drenaje lineal o una superficie tributaria, manifestándose transferencias en sus divisorias, con pluralidad de puntos de salida en algunos casos, y dada la insuficiente pendiente las respuestas a los estímulos pluviales se dan en términos de acumulación. Son los denominados Sistemas Hidrológicos No Típicos (SHN).

La morfología de estos últimos sistemas condiciona los procesos hidrológicos y el modelo conceptual requerido para describirlos (Fertonani y Prendes 1983, Kovacs 1983, Tricart 1983, Caamaño Nelli y Zimmermann 1990, Kruse y Zimmermann 2002). Hay un aspecto clave en el funcionamiento hidrológico de esos sistemas que es la marcada interacción de la hidrología superficial con la subterránea.

En función de los niveles freáticos los excedentes hídricos pueden incorporarse al perfil del suelo o generar derrames y acumulación superficial de consideración. La zona no saturada (ZNS) representa la interfaz entre los procesos superficiales y subterráneos, y la estimación correcta de los flujos de intercambio entre ambos ambientes a través de ella es crucial a la hora de realizar simulaciones mediante modelos hidrológicos en áreas de llanura. Como una alternativa aplicable a estos sistemas se han desarrollado esquemas de modelación, basados en celdas interconectadas representativas de los ambientes que intercambian materia, energía e información. Un ejemplo de esto es el modelo de Simulación Hidrológica de Áreas de Llanura, SHALL (Zimmermann y Riccardi 2000).

El modelo es hidrodinámico cuasi-3D, que contempla las componentes subterráneas y superficiales de flujos de agua, conjuntamente con los flujos verticales hacia el acuífero y la atmósfera.
En cada celda ó unidad de discretización espacial, el modelo SHALL puede cuantificar dinámicamente variables de estado (almacenamientos por intercepción, superficial, en el perfil del suelo y subterráneo) y flujos de intercambio (evapotranspiración, ascenso capilar, escurrimiento superficial, mantiforme y encauzado, a superficie libre y a través de constricciones -puentes, alcantarillas, sobrepaso de terraplenes, etc.-, infiltración, percolación profunda y escurrimiento subterráneo).La aptitud de conectar la hidrología de superficie con la subterránea, lo habilita para realizar predicciones acerca de evoluciones en los procesos hidrológicos provocados por acciones antrópicas a escala de cuenca y en el largo plazo.

El modelo ha sido aplicado en sistemas abiertos del sur santafesino (Zimmermann y Riccardi 2003, Méndez Zacarías y Zimmermann 2011) y sistemas cerrados pampeanos (Zimmermann y Mecca 2010). Actualmente se prevé la aplicación del modelo en un sector deprimido dela cuenca del arroyo del Azul, que conforma la vertiente sur del río Salado (provincia de Bs. As.). Es aquí donde los niveles freáticos se manifiestan someramente otorgando una particularidad a la modelación de cierta complejidad.

Con fines de adaptación a esta realidad es que se ha propuesto un esquema numérico novedoso para el flujo de intercambio superficial-subterráneo para áreas de niveles freáticos someros, el cual se denominó 4C (cuatro celdas). El esquema propuesto contempla la heterogeneidad de la ZNS y, a su vez, presenta simplicidad y rápida resolución en términos de tiempo computacional. Este nuevo esquema numérico se ha incorporado en la estructura del modelo hidrológico-hidrodinámico SHALL.

Es el propósito de este trabajo comparar el esquema $4 \mathrm{C}$ con estimaciones realizadas por otros modelos de flujo en ZNS de reconocida trayectoria como el HYDRUS-1D (Simunek et al., 1998) para ejemplos propuestos y analizar su posible performance en la aplicación del modelo en las áreas deprimidas del arroyo del Azul.

\section{AREA DE ESTUDIO}

La cuenca del arroyo del Azul se ubica en la zona central de la provincia de Buenos Aires, entre los meridianos $58^{\circ} 51^{\prime}$ y $60^{\circ} 10^{\prime}$ de longitud oeste y paralelos $36^{\circ} 09^{\prime}$ y $37^{\circ} 19^{\prime}$ de latitud sur (Figura 1). 
Abarca una superficie de $6237 \mathrm{~km}^{2}$ y como formas destacables se reconocen un sub-ambiente serrano hacia el sur de la cuenca con altitudes por sobre los $200 \mathrm{msnm}$ (pendiente media del terreno del $5 \%$ ), y un sub-ambiente de llanura hacia el norte, por debajo de los $130 \mathrm{msnm}$ (con pendientes que varían entre 0.5 y $0.8 \mathrm{~m} / \mathrm{km})$.
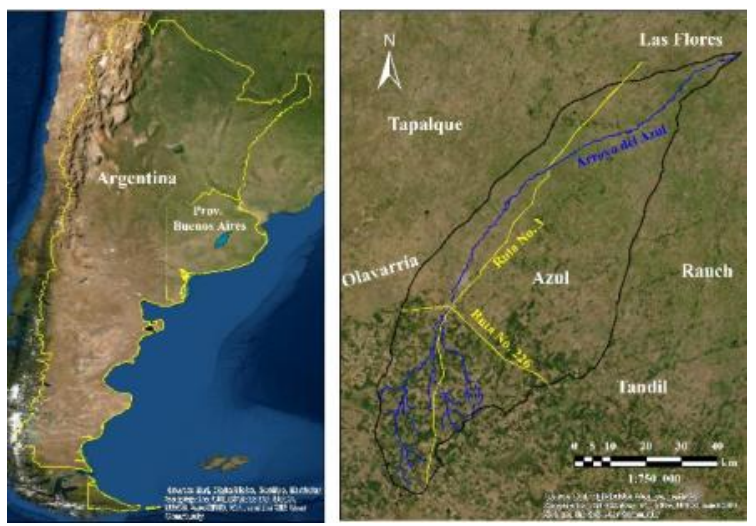

Figura 1. Ubicación relativa de la cuenca del arroyo del Azul, y principales localidades y vías de comunicación.

Entre ambos ambientes se desarrolla una zona de transición, caracterizada por suaves ondulaciones.

En el sector más bajo, sumamente llano, con sus suelos nátricos y drenaje deficiente, se desarrolla principalmente la cría ganadera extensiva en una matriz de pastizales naturales interrumpida por las numerosas cubetas y lagunas de carácter mayoritariamente semi-permanente y por dunas parabólicas y longitudinales (Entraigas et al., 2019).

Desde el punto de vista hidrológico, lo más notable del comportamiento del agua en cuencas tan deprimidas como la del Azul es la acumulación del agua sobre la superficie, y la interacción que se establece entre las aguas superficiales y las subterráneas, conformando un Sistema Hidrológico No Típico. Específicamente se ha concentrado el interés en la modelación de un sector del área más deprimida de la cuenca (Figura 2).

El dominio de modelación consiste en un rectángulo de $24 \mathrm{~km}$ en dirección SO-NE y $29 \mathrm{~km}$ en dirección NO-SE, el que se encuentra en su mayor parte incluido en la cuenca superficial del arroyo del Azul. Para cumplimentar con los fines comparativos es necesario caracterizar físicamente el medio que constituye la ZNS.

Una característica marcada del área de estudio es la presencia de niveles freáticos muy someros (50-100 $\mathrm{cm}$ de profundidad) en casi su totalidad, y durante gran parte del año.

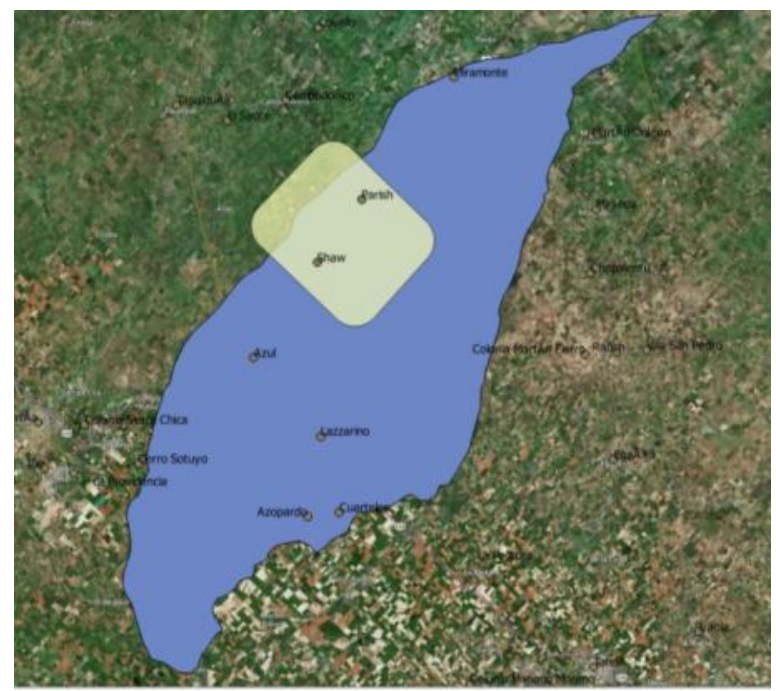

Figura 2. Cuenca del arroyo del Azul y área de interés para la modelación

Estos dos escenarios, características físicas del medio y presencia de napa somera, marcan las condiciones que deben respetarse en las simulaciones a comparar.

\section{Caracterización Edafológica}

Para este propósito se contó con información elaborada por el Instituto Nacional de Tecnología Agropecuaria (INTA) la cual fue cartografiada digitalmente obteniendo un mapa con asociaciones de perfiles típicos de suelos. En el área de estudio se detectaron 18 series de suelos que se combinan dando lugar a 23 asociaciones, de las cuales se seleccionaron las más representativas para este estudio. En cada perfil de las series, en función de su profundidad y su estratigrafía, se detallan en las cartas de INTA entre 3 y 8 horizontes de los cuales se ha publicado la composición textural, junto a otros parámetros tales como contenido de materia orgánica, contenido de carbono, capacidad de intercambio catiónico, $\mathrm{pH}$, etc.Con esta información, aplicando funciones de pedotransferencia de reconocida representatividad en la región pampeana (Zimmermann y Basile 2008, 2011, 2014) se obtuvieron los parámetros hidráulicos respectivos para las series de suelos en las simulaciones. Los parámetros hidráulicos de la ZNS que se estimaron son los siguientes: conductividad hidráulica saturada; potencial de entrada de aire y conectividad de poros para la curva de retención (modelo de Brooks-Corey) y humedades volumétricas 
residuales y de saturación. En primer lugar se estimaron los parámetros medios para cada serie de suelo en toda su profundidad y luego en función de ellos se valoraron los parámetros para cada asociación ó consociación de suelos ponderando con la fracción de series de suelos que la componen (Figura 3).

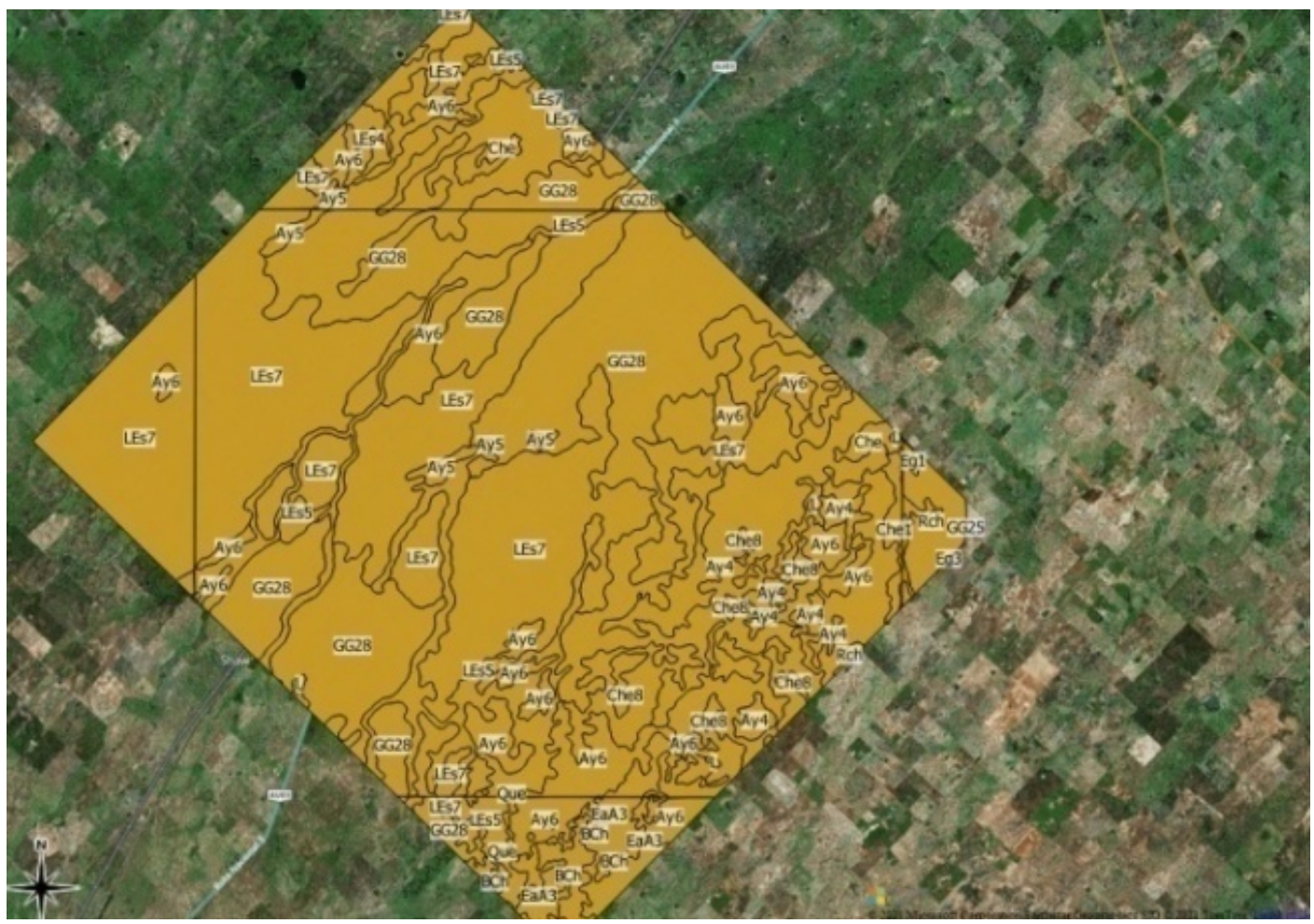

Figura 3. Mapa de ubicación de asociaciones suelos en el área de estudio

Para estimar humedades características se emplearon las funciones propuestas por Vereecken et al. (1989), para densidad aparente la fórmula de Tomasella y Hodnett (1998), para el potencial de entrada de aire la fórmula de Wösten et al. (1999) y las conductividades hidráulicas saturadas la fórmula de Saxton et al. (1986).

En todos los casos se consideró discriminar los parámetros por horizontesedáficos con el propósito de caracterizar la heterogeneidad del perfil.

\section{MODELO DE FLUJOS 4C PROPUESTO PARA LA ZONA NO SATURADA.}

El modelo está basado en la ecuación de Richards, para estimar la redistribución de humedad en la ZNS y los montos de agua intercambiados con la atmósfera y el acuífero. Solamente está contemplado el flujo en la dirección vertical. La resolución de la ecuación de continuidad (ecuación 1) se realiza finalmente en términos del contenido volumétrico de humedad, $\theta$,para conocer una de las variables del balance hidrológico de manera directa aunque la ecuación de flujo se plantea en términos del potencial mátrico, $\psi$, (ecuación 2):

$$
\begin{aligned}
& \frac{\partial \theta}{\partial t}=-\frac{\partial q}{\partial z} \\
& q=-K\left(\frac{\partial \psi}{\partial z}+1\right)
\end{aligned}
$$

donde q es la velocidad de Darcy del flujo no saturado, $\theta$ el contenido volumétrico de humedad, $\mathrm{z}$ la coordenada vertical (positiva hacia arriba), $K(\theta)$ la conductividad hidráulica no saturada, $\psi(\theta)$ el potencial mátrico o potencial de succión.

El medio poroso no saturado se representa en forma discreta mediante un conjunto de 4 celdas que se extienden verticalmente desde la superficie hasta el nivel freático $\mathrm{NF}$, tres celdas representativas de cada horizonte edáfico (A,B y C) y la última celda sumergida en el acuífero freático la cual representa la condición de borde inferior (Figura4). 


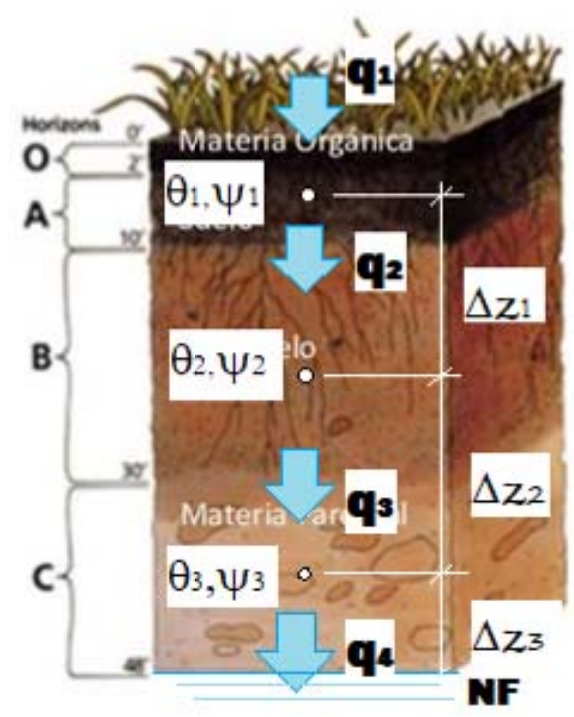

Figura 4. Esquema de discretización de la ZNS

Los 3 horizontes edáficos están presentes en la zona de estudio (caracterizada por suelos molisoles). Cada celda (horizonte) con su contenido de humedad $\theta_{\mathrm{i}}$, potencial mátrico $\psi_{\mathrm{i}}$ y sus intercambios de flujo $\mathrm{q}_{\mathrm{i}}$ determinados, separadas por distancias variables $\Delta \mathrm{z}_{\mathrm{i}}$ según las asociaciones de suelos que las caracterizan. Para la resolución de las ecuaciones (1) y (2) se ha propuesto un esquema numérico de tipo explícito en diferencias finitas. El esquema es centrado en el espacio y progresivo en el tiempo. En los bordes de celdas se evalúan los flujos de intercambio y en los centros de celdas se estiman las humedades y potenciales:

$\theta_{j}^{n+1}=\theta_{j}^{n}-\frac{2 \Delta t}{\Delta z_{j}+\Delta z_{j+1}}\left(q_{j}^{n}-q_{j+1}^{n}\right) ; j=1, j f$

$\mathrm{q}_{\mathrm{j}}^{\mathrm{n}}=-\mathrm{K}_{\varepsilon}\left(\frac{\psi_{\mathrm{j}-1}^{\mathrm{n}}-\psi_{\mathrm{j}}^{\mathrm{n}}}{\Delta \mathrm{z}_{\mathrm{j}-1}}+1\right) ; \mathrm{j}=2, \mathrm{j} \mathrm{f}+1$

donde $\mathrm{j}$ y $\mathrm{n}$ representan los índices de discretización espacial y temporal, respectivamente, mientras que jf es un indicador de la celda que alcance el nivel freático. Como valor "representativo" de la conductividad hidráulica no saturada $\mathrm{K}_{\varepsilon}$ se propone aquí:

$\mathrm{K}_{\varepsilon}=\varepsilon \mathrm{K}_{\min }+(1-\varepsilon) \mathrm{K}_{\max }$

donde $\mathrm{K}_{\min } \quad \mathrm{y} \quad \mathrm{K}_{\max }$ son las conductividades hidráulicas no saturadas mínima y máxima entre los estratos contiguos, respectivamente, y $\varepsilon$ un coeficiente de ponderación $(0<\varepsilon<1)$. De esta manera, $\varepsilon$ pondera el peso del estrato menos permeable en el valor del flujo no saturado.

La secuencia de cálculo es la siguiente: (a) Partir de una condición inicial de humedades $\theta_{\mathrm{j}}^{0}$ en el perfil, (b) resolver la ecuación de flujo (ecuación 4) para las celdas no saturadas, (c) resolver la ecuación de continuidad (ecuación 3) determinando las humedades del perfil en el siguiente paso de tiempo y (d) retomar paso (b) hasta tiempo de finalización de la simulación.

Para las condiciones de contorno, se plantea en el borde superior una condición de flujo establecida balanceando la capacidad de infiltración y la intensidad de precipitación:

$\mathrm{q}_{1}^{\mathrm{n}}=\max \left[-\mathrm{K}_{\varepsilon}\left(\frac{-2 \psi_{1}^{\mathrm{n}}}{\Delta \mathrm{z}_{1}}+1\right) ;-\mathrm{i}^{\mathrm{n}}\right]$

donde $i^{\mathrm{n}}$ es la intensidad de lluvia efectiva, estimada como la intensidad de precipitación (lámina caída sobre intervalo de tiempo) que no es interceptada por la vegetación. El primer término de la ecuación (6) representa la capacidad de infiltración y el segundo la disponibilidad de agua para infiltrarse.

Como condición de borde inferior se considera la última celdaincluida en la capa acuífera con humedad de saturación y potencial mátrico respectivo igual a cero:

$\theta_{\mathrm{jf}}^{\mathrm{n}}=\theta_{\mathrm{s}} ; \quad \psi_{\mathrm{jf}}^{\mathrm{n}}=0$

Deben conocerse parámetros del suelo tales como las curvas de conductividad hidráulica $K(\theta)$, y de retención de humedad $\psi(\theta)$. En el presente trabajo se han adoptado las relaciones de Brooks-Corey cuyos parámetros para cada horizonte $\mathrm{y}$ asociación de suelos analizada se han obtenido mediantes ecuaciones de pedotransferencia, según se ha detallado previamente. Los lazos de histéresis entre secado y mojadura no se han tenido en cuenta.

Este submodelo de flujos en la ZNS se ha incorporado en el contexto del modelo de simulación SHALL que se ha descripto en la introducción. 


\section{MODELO DE REFERENCIA HYDRUS-1D}

HYDRUS-1D, es un software diseñado par la simulación del movimiento de solutos, calor y agua en un medio unidimensional con saturación variable (Simunek et al., 1998). El programa HYDRUS resuelve numéricamente la ecuación de Richards para el flujo de agua en medio no saturado y las ecuaciones de tipo convección-dispersión para el transporte de calor y solutos. Las versión empleada incorpora un término de sumidero para tener en cuenta la absorción de agua por las raíces de las plantas, también puede considerar porosidad dual con una fracción del contenido de agua móvil y fracción inmóvil, aunque en los ejemplos que se analizaron no se emplearon estos complementos. La región de flujo puede estar compuesta por suelos heterogéneos o estratificados.

La parte de flujo de agua del modelo puede tratar con límites prescritos de altura y flujo, límites controlado por las condiciones atmosféricas, así como por las condiciones de frontera de drenaje libre. Las ecuaciones de flujo y transporte que gobiernan se resuelven numéricamente usando elementos finitos lineales tipo Galerkin. HYDRUS también incluye una optimización de parámetros de reacción y transporte de solutos y/o hidráulicos del suelo mediante un algoritmo de tipo MarquardtLevenberg para la estimación inversa de los mismos.

El programa original se ha complementado con un menú de interfase gráfica para facilitar la carga de datos e interpretación de resultados (Simunek et al., 2005). El programa ha sido ampliamente utilizado en la solución de problemas de flujo y transporte de la ZNS y constituye un software de referencia mundial.

\section{ENSAYOS REALIZADOS}

\section{Descripción general}

El objetivo primordial, y sobre el cual se hará hincapié en las comparaciones, es lograr una satisfactoria estimación de la percolación acumulada (al final de cada ensayo) del perfil bajo diversas situaciones en sus contornos. Esto se ve justificado sobre la idea que el esquema propuesto para el flujo en la ZNS representa la interfase entre hidrología de superficie y subterránea en el modelo SHALL, por ende es importante una buena representación de los flujos de intercambios con ambos ambientes, por encima de una buena representación de la distribución de los contenidos de humedad en el perfil de suelo.

Además, dado que la composición textural de los perfiles de suelo tienen un importante contenido de arcilla y los flujos se preven lentos, se propusieron simulaciones en el largo plazo para contemplar la llegada desde la superficie al nivel freático.

Las simulaciones sólo se realizaron para el flujo hidráulico unidimensional, con tres capas representativas de la heterogeneidad del perfil (horizontes edáficos), sin considerar porosidad dual ni histérisis en las curvas de conductividad hidráulica y de retención de humedad.

La condición de borde superior consistió en flujo establecido para el primer ensayo y una serie de eventos de precipitación a lo largo de un período de simulación, como condición variable en el tiempo, en el segundo.

La condición de borde inferior consistió en un valor de potencial mátrico preestablecido(nulo) a la profundidad coincidente con el nivel freático adoptado en cada ensayo.

La condición inicial se propuso con el perfil de humedad en equilibrio, es decir, $\psi(\mathrm{z})=-\mathrm{Z}_{\mathrm{NF}}-\mathrm{Z}$, donde $\mathrm{z}$ es la coordenada vertical ( $\mathrm{z}=0$ en la superficie y el sentido positivo del eje $z$ es ascendente).

Para las simulaciones con el modelo HYDRUS1D, sediscretizó cada perfil de suelo con intervalos uniformes de $5 \mathrm{~cm}$ para poder analizar el flujo a través de la ZNS, comenzando por el nodo 1 desde la superficie y avanzando en profundidad hasta la freática.

Se adoptaron los pasos máximos y mínimos de tiempo sugeridos por el programa para simulación numérica, así como también para los criterios de iteración y control de paso de tiempo.

Las simulaciones con el esquema de 4 celdas propuesto se hicieron bajo las mismas funciones de curva de retención y condiciones de borde propuestas para HYDRUS. La discretización del perfil consistió en hacer coincidir con los baricentros de cada perfil edáfico con los nodos de simulación de los perfiles de humedad y potencial mátrico, mientras que el nodo de condición de flujo inferior se seleccionó en función de la profundidad freática, variable en cada ensayo. 
Como el esquema propuesto es de tipo explícito, se verifica en cada momento de simulación la condición de Courant.

En principio, de las 23 asociaciones de series de suelos presentes área de estudio, descritas preceden-temente, se seleccionaron 14 representativas para realizar los ensayos comparativos. Los diferentes perfiles edáficos se grafican esquematicamente en la Figura 5.

La composición textural general de los perfiles es franca, con un tercio de cada fracción (arena, limo y arcilla). Los horizontes A son predominantemente francos-franco arenosos con un $23 \%$ de arcilla, $37 \%$ de limo y $40 \%$ de arena en términos medios. Los horizontes B son predominantemente franco arcillosos, con un 44\% de arcilla, 31\% de limo y $25 \%$ de arena. En los horizontes $\mathrm{C}$ con frecuencia hay presencia de tosca (cementado con carbonatos).

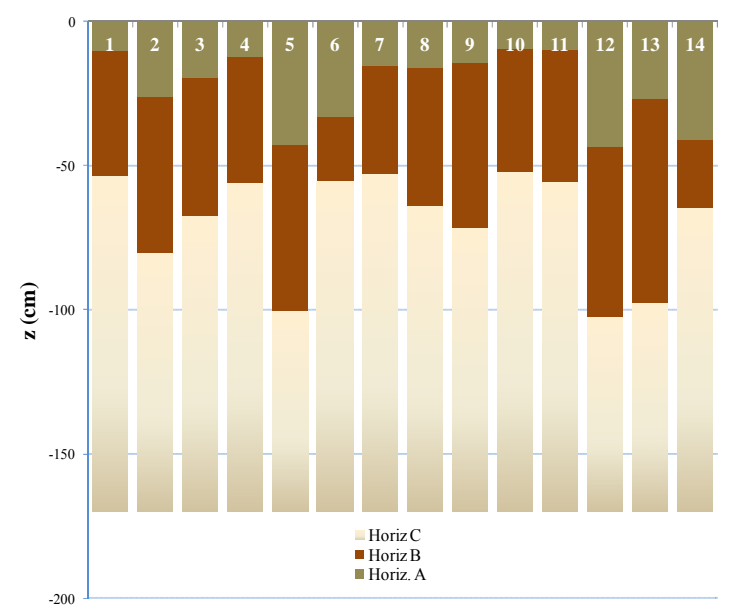

Figura 5. Esquema de los perfiles de suelo analizados

En Tabla 1, se resumen las principales características de los 14 perfiles edáficos analizados, el símbolo de la asociación respectiva cuya distribución geográfica se muestra en la Figura 3, la serie principal del perfil de suelos dominante, el nodo que representa la profundidad de cada horizonte, las propiedades hidráulicas de cada estrato y los nodos de observación que coinciden con los baricentros del esquema de 4 cedas simplificado que se propone como alternativa.

\section{Cuantificación de las comparaciones}

Para cuantificar el grado de cercania entre las dos estimaciones de la percolación (HYDRUS y esquema 4C) se consideraron, a saber: el coeficiente de eficiencia de Nash-Sutcliffe (NSE), el error medio cuadrático (RMSE), el sesgo (BIAS) y un error porcentual (EP) definidos bajo las siguientes formulaciones:

$$
\begin{aligned}
& \text { NSE }=1-\frac{\sum_{i=1}^{N}\left(R_{4 C}^{i}-R_{H y}^{i}\right)^{2}}{\sum_{i=1}^{N}\left(R_{H y}^{i}-R_{H y}^{m e d}\right)^{2}} \\
& R M S E=\sqrt{\frac{\sum_{i=1}^{N}\left(R_{4 C}^{i}-R_{H y}^{i}\right)^{2}}{N}} \\
& B I A S=\frac{\sum_{i=1}^{N}\left(R_{4 C}^{i}-R_{H y}^{i}\right)}{N} \\
& E P=100 \frac{B I A S}{R_{H y}^{m e d}}
\end{aligned}
$$

donde $\mathrm{N}$ es el número de ensayos realizados, $\mathrm{R}_{4 \mathrm{C}}^{\mathrm{i}} \mathrm{y}$ $\mathrm{R}_{\mathrm{Hy}}^{\mathrm{i}}$ representan las recargas acumuladas al nivel freático realizadas con el esquema de 4 celdas e HYDRUS-1D en el ensayo $i$, respectivamente $y$ $\mathrm{R}_{\mathrm{Hy}}{ }^{\text {med }}$ es el promedio de recargas acumuladas obtenidas mediante HYDRUS-1D para todos los ensayos.

Se consideró el coeficiente de ponderación $\varepsilon$ variable entre 0 y 1 , calculando las recargas $R_{4 C}$ para distintos $\varepsilon$ variando con intervalos de 0.05 , y evaluando los estadísticos de ajuste para cada ensayo.

\section{RESULTADOS}

\section{Ensayo 1: Intensidad de lluvia constante durante 10 días}

Una primer simulación se propuso con un flujo de baja intensidad constante de $0.5 \mathrm{~mm} / \mathrm{h}$ durante 10 dias, como condición de borde superior, lo que resulta en una superficie casi permanentemente encharcada.

En el borde inferior se propusieron diferentes profundidades freáticas, a saber: $2 \mathrm{~m}, 1.5 \mathrm{~m}, 1 \mathrm{~m}$ y 50 $\mathrm{cm}$, todas representativas del sector de estudio. No se consideraron profundidades mayores porque, para el tiempo de simulación propuesto, el flujo no las alcanzaría dadas las bajas permeabilidades de los estratos iluviales (horizonte B), principalmente. 
Tabla 1. Características de los perfiles edáficos analizados

\begin{tabular}{|c|c|c|c|c|c|c|c|}
\hline Perfil $N^{\circ}$ & Nodos'estrato & $\theta_{\mathrm{r}}$ & $\theta_{5}$ & $\alpha$ & $\mathrm{n}$ & Ks & Nodos Obser. \\
\hline & & & & $\mathrm{l} / \mathrm{cm}$ & & $\mathrm{cmh}$ & \\
\hline 1 & 2 & 0.02 & 0.45 & 0.067 & 0.33 & 0.94 & 1 \\
\hline LEs 7 & 11 & 0.08 & 0.39 & 0.030 & 0.33 & 0.12 & 6 \\
\hline La Escocia & 22 & 0.05 & 0.41 & 0.048 & 0.33 & 0.43 & 17 \\
\hline 2 & 5 & 0.03 & 0.43 & 0.091 & 0.33 & 0.68 & 3 \\
\hline Ay6 & 16 & 0.08 & 0.39 & 0.032 & 0.33 & 0.15 & 11 \\
\hline Ayacucho & 30 & 0.03 & 0.43 & 0.091 & 0.33 & 0.68 & 23 \\
\hline 3 & 4 & 0.03 & 0.43 & 0.091 & 0.33 & 0.68 & 2 \\
\hline $\mathrm{GG} 28$ & 13 & 0.08 & 0.39 & 0.032 & 0.33 & 0.15 & 9 \\
\hline Gral Guido & 28 & 0.03 & 0.43 & 0.091 & 0.33 & 0.68 & 21 \\
\hline 4 & 3 & 0.03 & 0.43 & 0.091 & 0.33 & 0.68 & 1 \\
\hline Che & 11 & 0.08 & 0.39 & 0.031 & 0.33 & 0.13 & 7 \\
\hline Chelforó & 26 & 0.03 & 0.43 & 0.077 & 0.33 & 0.62 & 19 \\
\hline 5 & 9 & 0.03 & 0.42 & 0.067 & 0.33 & 0.70 & 4 \\
\hline $\mathrm{Eg}$ & 20 & 0.08 & 0.39 & 0.033 & 0.33 & 0.20 & 14 \\
\hline Egaña & 29 & 0.05 & 0.41 & 0.056 & 0.33 & 0.46 & 24 \\
\hline 6 & 7 & 0.04 & 0.41 & 0.071 & 0.33 & 0.80 & 3 \\
\hline Eg1 & 11 & 0.09 & 0.39 & 0.030 & 0.33 & 0.10 & 9 \\
\hline Egaña & 27 & 0.05 & 0.38 & 0.050 & 0.33 & 0.90 & 19 \\
\hline 7 & 3 & 0.03 & 0.43 & 0.091 & 0.33 & 0.68 & 2 \\
\hline Rch & 11 & 0.09 & 0.39 & 0.029 & 0.33 & 0.09 & 7 \\
\hline Ravch & 26 & 0.04 & 0.43 & 0.071 & 0.33 & 0.61 & 18 \\
\hline 8 & 3 & 0.02 & \begin{tabular}{l|l|}
0.47 \\
\end{tabular} & 0.045 & 0.33 & 1.20 & 2 \\
\hline LEs5 & 13 & 0.08 & 0.39 & 0.028 & 033 & 0.07 & 8 \\
\hline La Escocia & 19 & 0.08 & 0.39 & 0.032 & 0.33 & 0.15 & 16 \\
\hline 9 & 3 & 0.03 & 0.44 & 0.077 & 0.33 & 0.78 & 1 \\
\hline Che1 & 14 & 0.08 & 0.39 & 0.030 & 0.33 & 0.10 & 9 \\
\hline Chelforó & 30 & 0.04 & 0.42 & 0.067 & 0.33 & 0.55 & 22 \\
\hline 10 & 2 & 0.02 & 0.45 & 0.071 & 0.33 & 0.84 & 1 \\
\hline Ches & 11 & 0.08 & 0.39 & 0.031 & 0.33 & 0.10 & 6 \\
\hline Chelfforó & 23 & 0.04 & 0.42 & 0.056 & 0.33 & 0.53 & 17 \\
\hline 11 & 2 & 0.02 & 0.49 & 0.048 & 033 & 132 & 1 \\
\hline LEs4 & 11 & 0.08 & 0.40 & 0.028 & 0.33 & 0.07 & 7 \\
\hline La Escocia & 18 & 0.09 & 0.39 & 0.029 & 0.33 & 0.11 & 14 \\
\hline 12 & 9 & 0.03 & 0.42 & 0.077 & 0.33 & 0.60 & 4 \\
\hline Ay 5 & 21 & 0.08 & 0.39 & 0.034 & 0.33 & 0.18 & 15 \\
\hline Ayacucho & 30 & 0.05 & 0.42 & 0.059 & 0.33 & 0.50 & 25 \\
\hline 13 & 5 & 0.06 & 0.42 & 0.042 & 0.33 & 0.56 & 3 \\
\hline $\mathrm{EaA3}$ & 20 & $\begin{array}{ll}0.07 \\
\end{array}$ & 0.40 & 0.029 & 0.33 & 0.09 & 12 \\
\hline Est. Aldecoa & 27 & $\begin{array}{ll}0.07 \\
\end{array}$ & 0.40 & 0.037 & 0.33 & 0.21 & 23 \\
\hline 14 & 8 & \begin{tabular}{l|l|}
0.07 \\
\end{tabular} & 0.33 & 0.036 & 0.33 & 0.43 & 4 \\
\hline $\mathrm{BCh}$ & 13 & 0.08 & 0.39 & 0.038 & 0.33 & 0.23 & 11 \\
\hline Blanca Chica & 22 & 0.08 & 0.35 & 0.032 & 033 & 030 & 18 \\
\hline
\end{tabular}

La combinación de escenarios entre condiciones de borde y materiales seleccionados para la simulación resultaron en 56 casos $(\mathrm{N}=56)$, de los cuales en 13 situaciones la solución con HYDRUS1D no alcanzó la convergencia y no fueron consideradas en las comparaciones.

Esto ocurrió para los casos de nivel freático somero (entre $1 \mathrm{~m}$ y $50 \mathrm{~cm}$ ), principalmente. Entre las causas comunes a este comportamiento, y posibles fuentes de inestabilidades en los casos de no convergencia, se encontraron: baja conductividad hidráulica del horizonte iluvial, gran contraste entre conductividades hidráulicas entre horizontes contiguos y la presencia somera del horizonte iluvial. Posiblemente estos inconvenientes puedan subsanarse en el HYDRUS mediante una discretización espacial mas detallada en los contactos entre materiales distintos, pero esto no fue analizado aquí.

El valor de $\varepsilon$ óptimo que reflejó los mejores NSE y menores errores RMSE y BIAS fue de 1.00. En los casos de nivel freático más profundo $(\mathrm{NF}=-2 \mathrm{~m}) \mathrm{se}$ obtuvieron buenos resultados también con $\varepsilon=0.95$.

La Figura 6 resume las concordancias encontradas con $\varepsilon=1$, y las diferencias encontradas entre ambos esquemas. El error porcentual EP en este caso fue del $19 \%$.

\section{Ensayo 2: Período de lluvias de 96 diás.}

La intención de este ensayo es analizar el comportamiento del esquema numérico simplificado bajo situaciones en las que será aplicado, tanto respecto al sistema hidrológico como a sus forzantes pluviales. Para construir la serie de lluvias características de la región bajo estudio se contó con información pluviométrica del período 2014-2018. Se realizó un estudio estadístico considerando precipitaciones y ocurrencias de lluvia en intervalos de clase de 96 días. Los resultados obtenidos fueron los soguientes: precipitación media cada 96 días $=$ $259 \mathrm{~mm}$ (desvío estándar $=84 \mathrm{~mm}$ ) y ocurrencia media de lluvias cada 96 días $=17$ días (desvío estándar $=7$ días).

Consecuentemente, se optó por construir una serie de precipitaciones con 4 períodos de $68 \mathrm{~mm}$ caidas en 4 días, separados entre sí con períodos diferentes, los que fueron seleccionados de manera arbitraria y no constantes (Figura 7). Esto se realizó con el fin de analizar el comportamiento de la recarga simulada bajo diferentes condiciones de humedad previas a cada tormenta.

Con el fin de simplificar el ensayo, se consideraron sólo 4 de los 14 perfiles edáficos numerados como: 3 , 6, 10 y 14 (Figura 5). Los mismos representan situaciones medias y extremas en cuanto a conductividades hidráulicas $\mathrm{y}$ profundidad del horizonte iluvial.

Dado que el tiempo de simulación de este nuevo ensayo es casi 10 veces el del anterior, se consideraron dos profundidades adicionales del nivel freático, las cuales se sumaron a los ensayos: $2.5 \mathrm{~m}$ y $3 \mathrm{~m}$. 


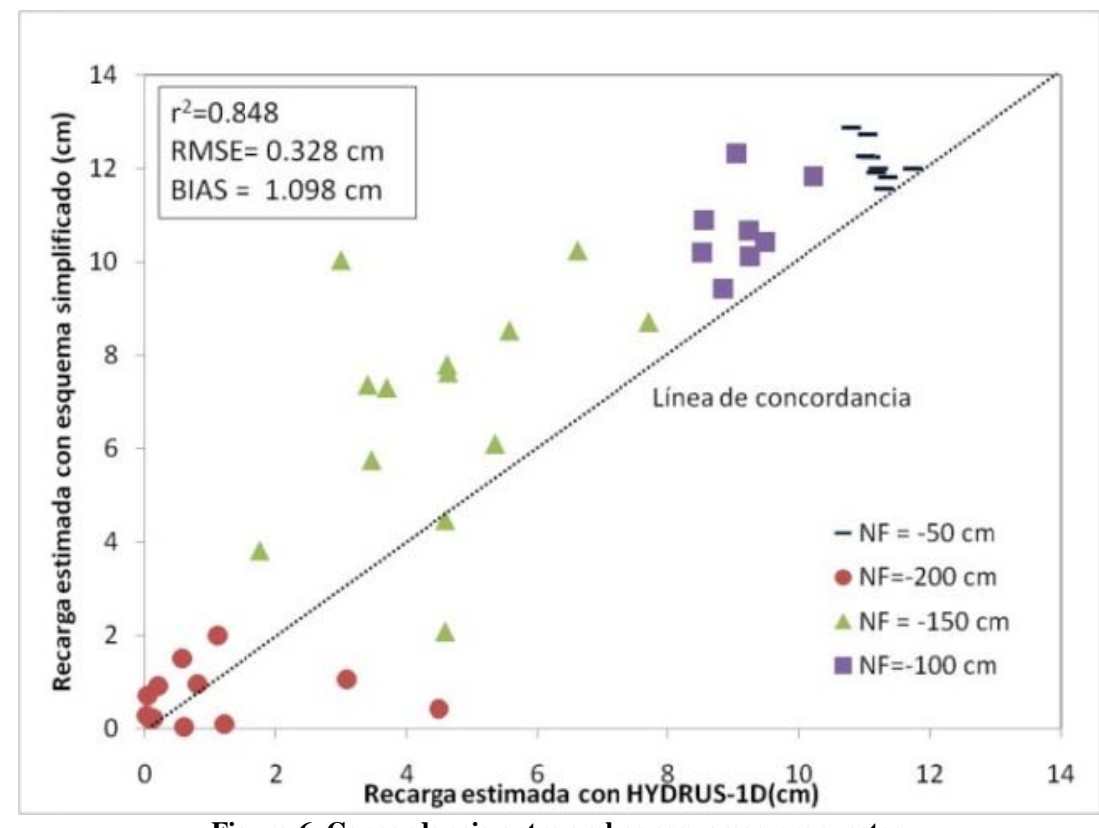

Figura 6. Concordancia entre ambos esquemas propuestos

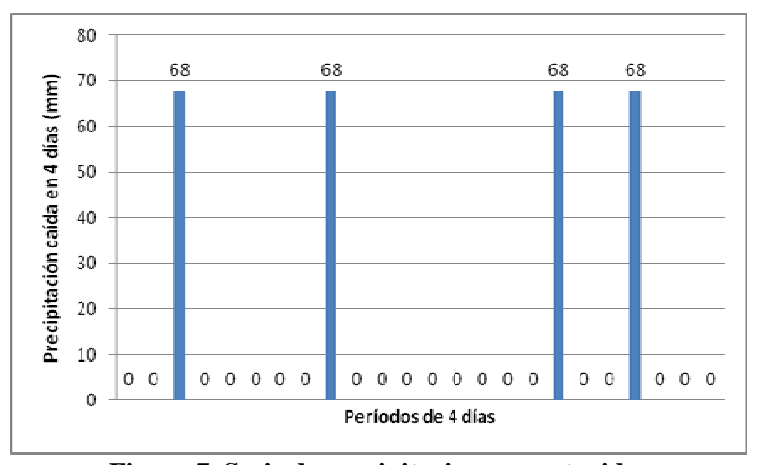

Figura 7. Serie de precipitaciones construida

Los valores óptimos del coeficientede ponderación de permeabilidades, $\varepsilon$, fueron diferentes para cada profundidad del nivel freático, pudiéndose encontrar una correlación lineal entre ambas variables. Por esta razón se propusieron expresiones para estimar $\varepsilon$ en función de la profundidad freática, a saber:

$$
\varepsilon=\left\{\begin{array}{cc}
\mathrm{PF}>150 \mathrm{~cm} & 0.7 \\
150 \mathrm{~cm}>\mathrm{PF}>100 \mathrm{~cm} & 1.6-0.6 \mathrm{PF} \\
\mathrm{PF}<100 \mathrm{~cm} & 1.0
\end{array}\right\}
$$

donde PF es a profundidad freática.

La Figura 8 resume las concordancias encontradas encontradas entre ambos esquemas, estimando $\varepsilon$ con las expresiones previas. El error porcentual EP en este caso fue del $1 \%$.
Aún cuando la función objetivo en las comparaciones ha sido las diferencias entre las recargas acumuladas al final de cada ensayo, se inspeccionaron las evoluciones de los contenidos volumétricos de humedad para cada perfil y horizonte edáfico considerado en ambos modelos. Pudo comprobarse una buena estimación de los perfiles de humedad en el tiempo por parte del esquema propuesto (Figura 9).

\section{DISCUSION}

Se han realizado dos ensayos para testear un esquema simple, explícito, de resolución numérica basado en diferencias finitas frente a otro de probada eficacia basado en un esquema de elementos finitos, de grilla variable, implícito iterativo (HYDRUS-1D) para modelar el flujo no saturado entre el nivel superficial y el freático.

El sistema a modelar está emplazado en una zona deprimida de la llanura bonaerense con elevada frecuencia de niveles freáticos a escasa profundidad.

El esquema simplificado propuesto forma parte de un modelo de flujo superficial y subterráneo cuasi 3D de celdas (SHALL) y surge la necesidad de validar su comportamiento en situaciones similares a las que operará, poniendo enfasis principalmente en el intercambio de flujo (recarga) entre ZNS y acuífero. 


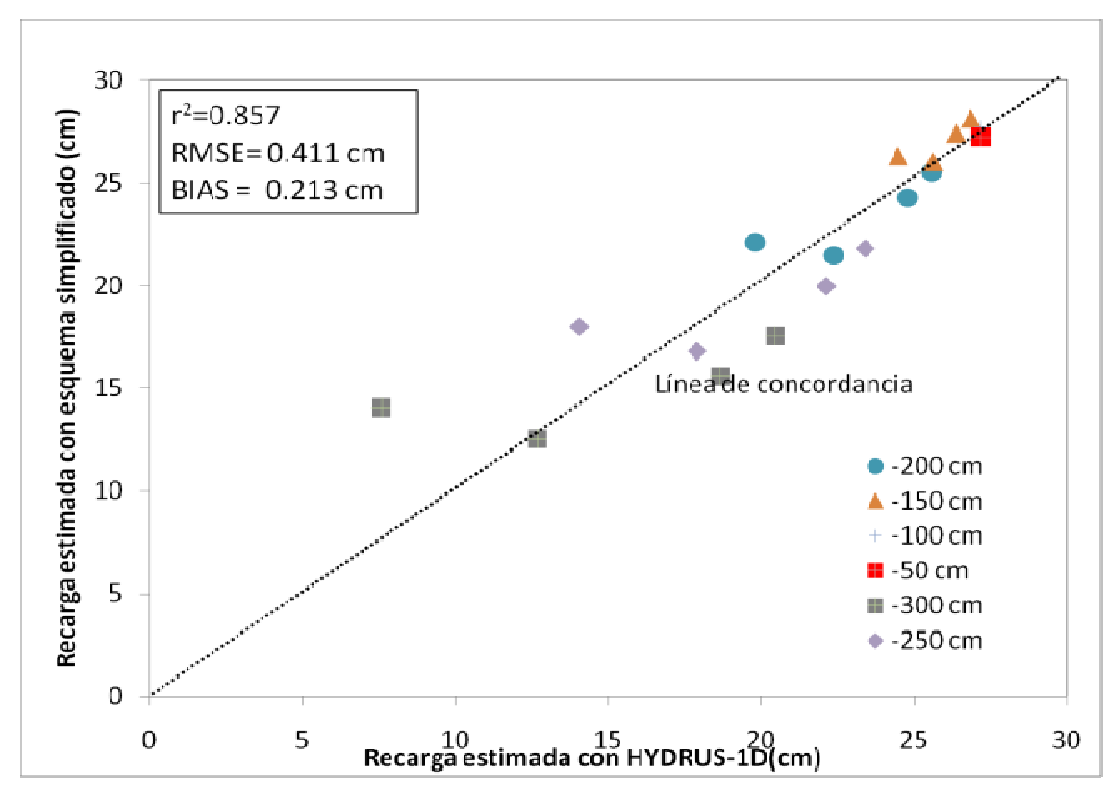

Figura 8. Concordancia entre ambos esquemas propuestos
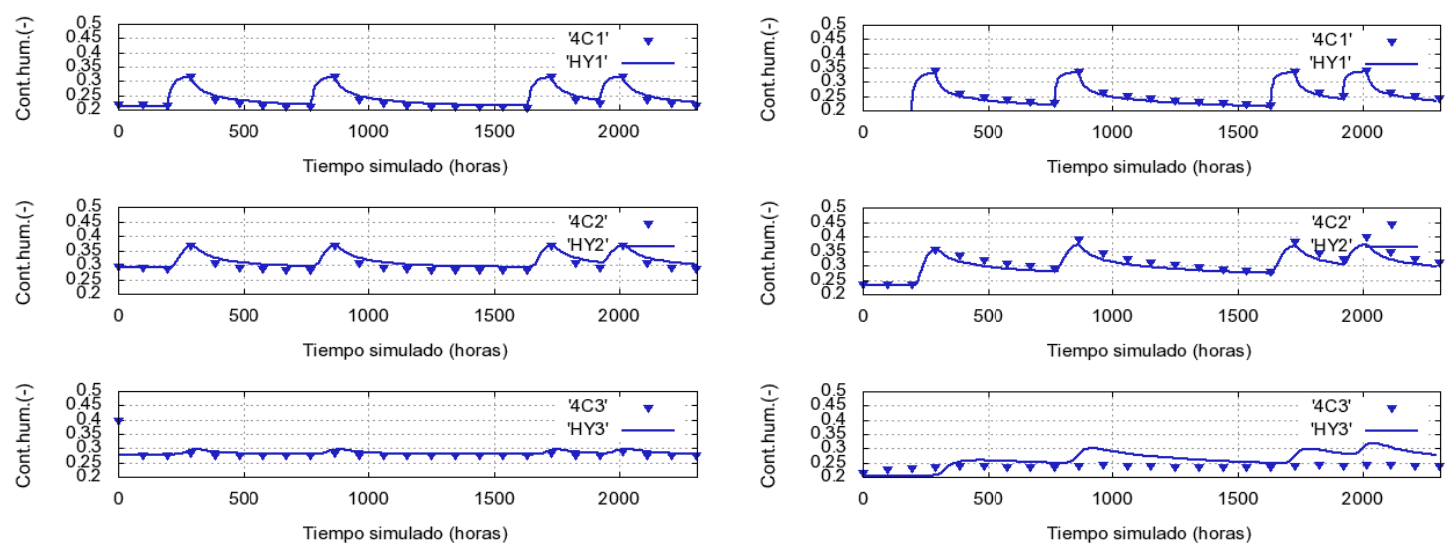

Figura 9. Evolución de contenidos de humedad en el segundo ensayo para los 3 horizones edáficos del perfil $\mathrm{N}^{\circ} 6$ y profundidad de napa $-150 \mathrm{~cm}$ (izquierda) y perfil No 10 profundidad de napa $-300 \mathrm{~cm}$ (derecha). Simulación con HYDRUS-1D (líneas continuas, HY1, HY2, HY3) y esquema 4C (triángulos, 4C1, 4C2, 4C3).

Se consideran 14 tipos de perfiles edádicos representativos de la zona, cuyas propiedades hidráulicas se han obtenido mediante funciones de pedotransferencia.

El primer ensayo propuesto para las comparaciones, implica una situación difícilmente alcanzada en la realidad, son 10 dias consecutivos de precipitación de baja intensidad, pero ha permitido conocer los comportamientos de los modelos a comparar en un escenario de saturación progresiva bajo niveles freáticos de someros a casi superficiales.

El comportamiento del esquema simplificado fue aceptable $\left(r^{2}=0.848\right.$, RMSE $\left.=0.328 \mathrm{~cm}\right)$, aunque con una tendencia a la sobreestimación de la recarga respecto al HYDRUS (BIAS $=+1.098 \mathrm{~cm}$ ).

Cabe mencionar que la propuesta del coeficiente de ponderación de permeabilidades de horizontes adyacentes tuvo como efecto darle el peso total al horizonte menos permeable, ya que el valor óptimo fue prácticamente la unidad (ecuación 5). En otras palabras, el horizonte iluvial controla el flujo en situaciones de saturación superficial y niveles freáticos poco profundos.

$\mathrm{Si}$ bien este ensayo representa una situación poco común, diferencias del $20 \%$ entre ambos esquemas muestran una expectativa no alcanzada. 
En el segundo ensayo, las condiciones del borde superior propuestas sí representan situaciones comunes a las que será sometido el esquema simplificado en el contexto de la modelación con el SHALL. De hecho, estas condiciones se han extraido de estadísticas de precipitación relativamente recientes.

Dado que la simulación se extiende a 96 dias, con tiempo suficiente para flujos más penetrantes, se incorporan dos situaciones adicionales de niveles freáticos más profundos como condiciones para el borde inferior.

Para estos casos el coeficiente de ponderación de permeabilidades encontró su valor óptimo en 0.7 , lo que implica una distribución del peso para las permeabilidades entre los estratos adyacentes, caso que se diferencia del ensayo anterior. Esto da la pauta que para situaciones donde el nivel freático es más profundo el flujo es comandado por las características hidráulicas de ambos horizontes adyacentes. Cuando el nivel freático es más somero, el flujo es comandado por el horizonte menos permeable, como sucede en el ensayo 1. Esta duali-dad de comportamiento justifica la propuesta de un coeficiente de ponderación vinculado al nivel freático, mediante una relación lineal, como se propuso en el ítem anterior. Esta propuesta mejoró sustancialmente el pronóstico de la recarga para el esquema simplificado $\left(r^{2}=0.857\right)$.

En este set de comparaciones los errores cuadráti-cos medios resultaron semejantes $($ RMSE $=0.411 \mathrm{~cm})$ y los sesgos muchos menores (BIAS $=+0.213 \mathrm{~cm}$ ). El error porcentual del $1 \%$ respecto al sesgo es bajo y hace que las comparaciones entre ambos esquemas sean similares.

Contemplando que este segundo ensayo se ajusta a condiciones reales y factibles para la zona de estudio se considera el desempeño del esquema simplificado propuesto como satisfactorio.

Además, si bien el objetivo propuesto para los ajustes era una estimación muy próxima en los valores de recarga final acumulada entre ambos esquemas, la estimación de las evoluciones de humedades en el perfil resultó muy similar. No obstante en las simulaciones con niveles freáticos más profundos(mayores incrementos $\Delta \mathrm{z}$ entre los últimos estratos en el esquema 4C), como se manifiesta en la Figura 9, derecha, se observa una distorsión en la estimación razón por la cual deberán analizarse estas situaciones con más detenimiento.
Estos dos elementos dan la pauta que el esquema simplificado propuesto para ser integrado en el modelo SHALL es un buen estimador de variables de estado y los flujos de intercambio en la ZNS.

\section{CONCLUSIONES}

Se propone un esquema numérico de diferencias finitas explícito de 4 celdas, físicamente basado, para representar el flujo no saturado y heterogéneo entre el nivel superficial y el freático. En este esquema y para la ecuación de flujo, basada en la ecuación de Richards, se propone una ponderación entre las conductividades hidráulicas de estratos adyacentes, que presentan contrastes marcados entre sí. El esquema es comparado con modelos de reconocida trayectoria como el HYDRUS-1D.

El sistema hidrológico a representar se emplaza en una zona deprimida de la llanura bonaerense con elevada frecuencia de niveles freáticos someros y presencia de un estrato arcilloso a poca profundidad.

Se realizan dos ensayos para testear la bondad del esquema, uno con intensidad de lluvia constante durante 10 días y otro con una serie de precipitaciones, estadísticamente representativas de la climatología local, extendida durante 96 dias. En ambos escenarios se consideraron las características físicas de los perfiles edáficos de la zona de estudio y la presencia somera de los niveles freáticos.

El primer ensayo representa un situación hipotética, difícilmente alcanzada en la realidad, pero ha permitido conocer las respuestas ante escenarios de saturación progresiva con niveles freáticos casi superficiales. La respuesta del esquema se considera aceptable con diferencias porcentuales del orden al 19\%.

En el segundo, el cual refleja situaciones estadísticamente posibles en cuanto a series climáticas, el esquema propuesto presentó un comportamiento satisfactorio, con diferencias porcentuales del $1 \%$ respecto al modelo HYDRUS-1D. Además, muestra un buen ajuste en las evoluciones de contenidos de humedad de cada estrato.

Se considera que estos resultados validan el esquema simplificado propuesto permitiendo una respuesta rápida en términos de tiempo computacional, haciéndolo apto para su inclusión en el modelo SHALL. 


\section{REFERENCIAS BIBLIOGRÁFICAS}

Caamaño Nelli, G. y Zimmermann, E. (1990) Tipología de los Sistemas Hidrológicos Superficiales. XVI Congreso Nacional Asoc. Arg. Geofis. Y Geod. Bahía Blanca. Argentina.

Entraigas, I., Vercelli, N., Fajardo, L. (2019). Plant communities along preferential superficial water flow paths across a floodplain landscape. Ecohydrology, 12(6), e2124. https://doi.org/10.1002/eco.2124

Fertonani, M., y Prendes, H. (1983) Hidrología en áreas de llanura. Aspectos Conceptuales Teóricos y Metodológicos. Coloquio de Hidrología de Grandes Llanuras. Olavarría.Argentina., 1, p 119-156.

Kovacs, G. (1983). General Principles of Flatlands Hydrology.Coloquio de Hidrología de Grandes Llanuras. Olavarría. Argentina, p 297-357.

Kruse, E. y Zimmermann, E. (2002). Hidrogeología de grandes llanuras. Particularidades en la llanura pampeana (Argentina).In: Workshop publication on Groundwater and Human development(pp. 20252038).

Méndez Zacarías J. y Zimmermann E. (2011). Uso De Sistemas De Información Geográfica Para Parametrización De Modelos De Simulación Hidrológica En Llanuras.XXIII Congreso Nacional del Agua y VI Simposio de Recursos Hídricos del Cono Sur. Resistencia. Argentina.

Saxton, K., Rawls,W., Romberger,J.,and Papendick R. (1986). Estimating generalized soil-water characteristics from texture.Geoderma, (102),275 297.

Simunek, J., Huang K., y Van Genuchten, M. (1998).The HYDRUS code for simulating the one dimensional movement of water, heat, and multiple solutes in variably saturated media. Version 6.0, Research Report No. 144, U.S. Salinity Laboratory, USDA, ARS, Riverside, California, 164pp.

Simunek, J., Van Genuchten, M. T., \& Sejna, M. (2005). The HYDRUS-1D software package for simulating the one-dimensional movement of water, heat, and multiple solutes in variably-saturated media. University of California-Riverside Research Reports, 3, 1-240.

Tricart, J. (1983) L'Hydrologie des Grans Plains, Quelques Reflexions Methodologiques. Coloquio de Hidrología de las grandes Llanuras.Olavarría, Argentina, 2, p 1191-1193.

Tomasella, J. y Hodnett, M. (1998). Estimating soil water retention characteristics from limited data in Brazilian Amazonia.Soil science,163(3), 190-202.

Vereecken, H., J. Maes, J. Feyen, and P. Darius(1989). Estimating the soil moisture retention characteristic from texture, bulk density, and carbon content. Soil
Science. 148, 389-403.

Wösten, J, A. Lilly, A. Nemes, and C. Le Bas (1999). Development and use of a database of hydraulic properties of European soils. Geoderma 90, 69-185.

Zimmermann E. y Riccardi , G. (2000)Modelo de simulación hidrológica superficial para áreas de llanura. XIX Congreso Latinoamericano de Hidráulica. Córdoba. Argentina.

Zimmermann, E. y Riccardi, G. (2003). Modelo hidrológico superficial y subterráneo desarrollado para la simulación de sistemas de llanura. 1. Aplicación en el sistema Ludueña (Santa Fe, Argentina). Boletín Geológico y Minero, 114(2), 147158.

Zimmermann, E. y Basile, P. (2008). Uso de funciones de pedotransferencia para la estimación de parámetros hidráulicos en suelos limosos (llanura Argentina). Boletín del Instituto Geológico Minero de España, 119(1), 71-80.

Zimmermann E. y J. Mecca (2010).Aplicación del modelo SHALL en los sistemas hidrológicos de las lagunas don Tomás y bajo Giuliani (La Pampa, Argentina) para la evaluación de impactos provocados por efluentes y escurrimientos. I Congreso Internacional de Hidrología de Llanuras. Azul, Buenos Aires, Argentina .

Zimmermann, E. y Basile, P. (2011). Estimación de parámetros hidráulicos en suelos limosos mediante diferentes funciones de pedotransferencia.Tecnología y ciencias del agua,2(1), 99-116.

Zimmermann, E. y Basile, P. (2014). Metodología de agregación para estimar conductividades hidráulicas en suelos heterogéneos insaturados.Tecnología $y$ ciencias del agua,5(4), 39-55.

\section{Tipo de Publicación: ARTICULO}

Trabajo recibido el 27/10/2021 y aprobado para su publicación el 29/11/2021.

\section{COMO CITAR}

Zimmerman, E. (2021). Esquema explícito para la solución numérica del flujo no saturado en medios heterogéneos bajo condición de niveles freáticos someros. Cuadernos del CURIHAM, 27,47-58. DOI: https://doi.org/10.35305/curiham.v27i.169

Este es un artículo de acceso abierto bajo licencia: Creative Commons Atribución - No Comercial Compartir Igual 4.0 Internacional (CC BY-NC-SA 4.0) (https://creativecommons.org/licenses/by-ncsa/4.0/deed.es) 